\title{
Development of an Early Prediction Model for Postoperative Delirium in Neurosurgical Patients Admitted to the ICU after Elective Craniotomy (E- PREPOD-NS): A Secondary Analysis of A Prospective Cohort Study
}

Hua-Wei Huang

Beijing Tiantan Hospital

Guo-Bin Zhang

Beijing Tiantan Hospital

Hao-Yi Li

Beijing Tiantan Hospital

Chun-Mei Wang

Beijing Tiantan Hospital

Yu-Mei Wang

Beijing Tiantan Hospital

Xiu-Mei Sun

Beijing Tiantan Hospital

Jing-Ran Chen

Beijing Tiantan Hospital

Guang-Qiang Chen

Beijing Tiantan Hospital

Ming Xu

Beijing Tiantan Hospital

Jian-Xin Zhou ( $\square$ zhoujx_ttyy@sina.com )

Beijing Tiantan Hospital https://orcid.org/0000-0003-4191-125X

\section{Research}

Keywords: Postoperative delirium, neurosurgical patients, intracranial surgery, risk prediction model, intensive care unit.

Posted Date: February 25th, 2021

DOI: https://doi.org/10.21203/rs.3.rs-271659/v1 
License: (c) (i) This work is licensed under a Creative Commons Attribution 4.0 International License. Read Full License 


\section{Abstract}

Background: Postoperative delirium (POD) is a significant clinical problem in neurosurgical patients after intracranial surgery. Identification of high-risk patients may optimise individual perioperative management, but an adequate and simple risk model for use at super early phase after operation has not been developed.

Methods: Adult patients were admitted to the ICU after elective intracranial surgery under general anaesthesia. The POD was diagnosed as Confusion Assessment Method for the ICU positive on postoperative day 1 to 3 . Multivariate logistic regression analysis was used to develop the early prediction model (E-PREPOD-NS) and the final model was validated with 200 bootstrap samples.

Results: Among 800 patients included in the study, POD occurred in 157 cases (19.6\%). We identified nine variables independently associated with POD in the final E-PREPOD-NS model: age $>65$ years [odds ratio $(\mathrm{OR})=3.336,95 \%$ confidence interval $(\mathrm{Cl})=1.765-6.305,1$ risk score point], education level $<9$ years (OR $=2.528,95 \% \mathrm{Cl}=1.446-4.419,1$ point $)$, history of smoking $(\mathrm{OR}=2.582,95 \% \mathrm{Cl}=1.611-4.140,1$ point $)$, history of diabetes $(\mathrm{OR}=2.541,95 \% \mathrm{Cl}=1.201-5.377,1$ point $)$, supra-tentorial lesions $(\mathrm{OR}=3.424,95 \% \mathrm{Cl}$ $=2.021-5.802,1$ point $)$, anesthesia duration $>360 \mathrm{~min}(\mathrm{OR}=1.686,95 \% \mathrm{Cl}=1.062-2.674,0.5$ point $), \mathrm{GCS}$ $<9$ at ICU admission (OR $=6.059,95 \% \mathrm{Cl}=3.789-9.690,1.5$ points), metabolic acidosis (OR $=13.903,95 \%$ $\mathrm{Cl}=6.248-30.938,2.5$ points $)$, and positioning of neurosurgical drainage tube $(\mathrm{OR}=1.924,95 \% \mathrm{Cl}=$ 1.132-3.269, 0.5 point). The area under the receiver operator curve (AUROC) of the risk score for prediction of POD was $0.865(95 \% \mathrm{Cl}=0.835-0.895)$. After internal validation by bootstrap, the AUROC was 0.851 $(95 \% \mathrm{Cl}=0.791-0.912)$. The model showed good calibration (Hosmer-Lemeshow $P=0.593)$.

Conclusions: The E-PREPOD-NS model based on nine perioperative risk factors can predict POD in patients admitted to the ICU after elective intracranial surgery with fairly good accuracy. External validation is needed before use in clinical practice.

\section{Background}

Postoperative delirium (POD) is a common postoperative complication which occurs in $11-51 \%$ in patients after major surgery (Inouye et al. 2014). Investigations have shown that POD is associated with significantly increased morbidity, mortality, intensive care unit (ICU) and hospital length of stays, healthcare costs and decreased postoperative neurocognitive dysfunctions (pNCD) (Franco et al. 2001; Abelha et al. 2013; Gleason et al. 2015; Evered et al. 2018). In a multicenter observational study, Van den Boogaard et al. found that neurosurgical patient was an independent risk factor for the development of ICU delirium (van den Boogaard et al. 2012). Therefore, there has been increased attention to the postoperative management of neurosurgical patients to improve long-term neurocognitive, and reducing POD has been identified as an important target for surgical quality improvement (Berian et al. 2018).

Around $30-40 \%$ of delirium cases are thought to be preventable (Siddiqi et al. 2006). Therefore, identifying high-risk patients for POD and early intervention for high-risk subjects may reduce the 
occurrence of delirium. Several prediction models for POD have been developed in different types of surgical patients (Kim et al. 2016; Kim et al. 2020; Bohner et al. 2003), but a risk model for POD specific to neurosurgical patients may provide unique insights in this vulnerable population. Recently, there are a few studies developed risk models to predict POD in neurosurgical patients, but each of these models has its own limitation, including small sample size, only involving single disease, or predictors unavailable at intensive care unit (ICU) admission (Wang et al. 2020b; Zhan et al. 2020; Harasawa et al. 2014; Flanigan et al. 2018). In this study, we developed, internally validated, and tested a risk score model for POD in neurosurgical patients using data from our previous prospective cohort study of adult patients after elective craniotomy (Wang et al. 2020a).

\section{Methods}

\section{Study Design and Data Source}

This was secondary analysis of our previous study (Wang et al. 2020a), a prospective cohort study of adult patients after elective intracranial surgery enrolled in the neurosurgical ICU in Beijing Tiantan Hospital, Capital Medical University, Beijing, China between March 2017 and February 2018 (ClinicalTrials.gov NCT 03087838).

We obtained written informed consent from all participants or their surrogates, who allowed for data abstraction from the electronic health record, and the study protocol was approved by the Institutional Review Board of Beijing Tiantan Hospital, Capital Medical University, Beijing, China (KY 2017-018-02).

\section{Participants}

Inclusion criteria for the present study were adult (at least $18 \mathrm{yrs}$ old) patients who underwent elective craniotomy under general anaesthesia admitted to ICU. Exclusion criteria were non-Chinese speaker, transsphenoidal surgery, cerebrospinal fluid shunt and drainage surgery, deep brain stimulation surgery, pre-operative coma (GCS $\leq 8$ ), pre-operative mental retardation due to Parkinson's disease or dementia, history of psychosis, could not be assessed for delirium, or unlikely to survive 24 hours.

\section{Outcome}

The primary outcome was the present of POD within 3 days after surgery. Delirium was determined by four researchers who have been trained to perform the delirium assessment. Delirium was evaluated by the Chinese version of the Confusion Assessment Method for the ICU (CAM-ICU) (Wang et al. 2013). The delirium assessment was performed in two steps. Firstly, the arousal level was assessed by RASS (Sessler et al. 2002). If RASS score $\leq-4$, the patient was recorded as comatose, the remaining delirium assessment was aborted. When the RASS score was greater than or equal to -3 , delirium was evaluated by the CAM-ICU. The CAM-ICU consists of four features: (1) acute onset of a mental status change or a 
fluctuating level of consciousness, (2) inattention, (3) disorganized thinking and (4) an altered level of consciousness. Delirium was diagnosed if both the first and second features were present, and either the third or fourth was present. During the study, delirium was assessed in each patient twice a day, at 08:00 to $10: 00$ and 20:00 to 22:00 at postoperative day 1 to 3 . The patients were assigned to the POD group if any assessment was diagnosed with delirium, otherwise they were assigned to the non-POD group.

\section{Predictors}

Candidate predictors of the primary outcome were obtained from the dataset of our previous prospective cohort study including demographic information, medical comorbidities, preoperative-related data, anaesthesia and surgery-related data and data at the time of ICU admission. Preoperative risk factors included preoperative of hydrocephalus, history of antiepileptic, lesion location (supra-tentorial lesion), and American Society of Anesthesiologists (ASA) physical status. Anaesthesia and surgery-related data included type of anesthesia (total intravenous anaesthesia, TIVA), intraoperative use of dexmedetomidine, intraoperative use of steroid, intraoperative transfusion and duration of surgery. Duration of surgery was categorized as $\leq 360 \mathrm{~min}$ and $\mathbb{3 6 0} \mathrm{min}$. Data at the time of ICU admission included Glasgow Coma Scale (GCS) at ICU admission, use of patient-controlled intravenous analgesia (PCIA), positioning of neurosurgical drainage tube (external ventricular drainage [EVD], subdural drainage, epidural drainage and drainage of operative cavity) and laboratory values (hemoglobin [ $\mathrm{g} / \mathrm{L}]$, serum sodium [mmol/L] and serum albumin [mmol/L]) and present of metabolic acidosis. Hemoglobin level less than $120 \mathrm{~g} / \mathrm{L}$ for women and less than $130 \mathrm{~g} / \mathrm{L}$ for men was defined as

anemia. Serum sodium $<135 \mathrm{mmol} / \mathrm{L}$ was defined as hyponatremia, serum albumin level $<3.5 \mathrm{~g} / \mathrm{dL}$ was defined as hypoalbuminemia and $\mathrm{pH}<7.35$ with bicarbonate $<24 \mathrm{mmol} / \mathrm{L}$ was defined as metabolic acidosis (Wassenaar et al. 2015).

\section{Statistical analysis}

Continuous variables were presented as mean \pm SD or median and interquartile range (IQR); categorical variables were expressed as frequency and percentage. Univariate analyses between the POD and the non- POD group were performed. The continuous variables used the Student $t$-test or Mann-Whitney Utest, while categorical variables were analyzed by the Pearson chi-square test or Fisher's exact test. Factors with $P$ values $<0.1$ in the univariate analyses were entered into the multivariate analysis with a stepwise backward logistic regression to identify independent risk factors for POD and developed a risk prediction model. The results of the final multivariate model are expressed as odds ratios (ORs) with their confidence intervals (Cls) and $p$ values. The absence of multicollinearity was verified by a variance inflation factor below 10 and interaction terms were not considered.

With the variables selected for the model, the probability of POD was derived from a logistic model with relative weights (regression coefficients) from each variable:

logit $=\mathrm{B}_{0}+\mathrm{B}_{1} \mathrm{x}_{1}+\mathrm{B}_{2} \mathrm{x}_{2}+\ldots$ 
$\mathrm{p}($ probability of postoperative delirium $)=1 /\left(1+\mathrm{e}^{-\left(\mathrm{B}_{0}+\mathrm{B}_{1} \mathrm{x}_{1}+\mathrm{B}_{2} \mathrm{x}_{2} \ldots\right)}\right)$.

The accuracy of the model was verified and compared in terms of calibration and discrimination. Calibration was evaluated using the Hosmer-Lemeshow goodness-of-fit test, $p$ values $>0.05$ indicated no significant difference between the observed and predicted outcomes. Discrimination was evaluated using the area under the receiver operator curve (AUROC) curve, and a value close to 1 indicates a greater probability that the outcome was predicted correctly in randomly chosen pairs of patients (Kadane et al. 2004; Omar 2010; Steyerberg et al. 2010). Because the performance in the development cohort may overestimate the performance of other patients, we validated the risk prediction score in the validation cohort by estimating discrimination and calibration. Internal validation was performed using bootstrapping with 200 repetitions (Omar 2010).

For ease of application, we turned the risk model into a risk prediction score. The coefficient was rounded to the closest whole number while conserving proportionality (Omar 2010). The sum of the scores obtained based on the presence or absence of the respective variables in each patient determined the probability of POD. Furthermore, to evaluate the ability of the model to predict increasing rates of POD (discrimination of model), patients were stratified by the prediction scores over the tertiles and divided into three ranges reflecting low, medium, and high risk for POD. Last, for analysis of diagnostic performance, information for sensitivity and specificity, positivity and negative predictive values, as well as positive and negative likelihood ratio, are presented.

Data analysis was performed using R v 3.6.3 (http:// www. R-project.org). For all analyses, two-side $P$ value of less than 0.05 was considered statistically significant.

\section{Results}

\section{Participant Characteristics}

All 800 patients who were admitted to the ICU after elective craniotomy were eligible for this cohort and were available for analysis. Of the analyzable cases, 157 (19.6\%) were POD patients and $643(80.4 \%)$ were non-POD patients. All cases were included in the development cohort to create the clinical score (EPREPOD-NS) for predicting POD.

\section{Development Cohort Analysis}

In the development cohort, univariate analysis for comparison of clinical and laboratory characteristic between POD and non-POD at the time of ICU admission was shown in Table 1. Compared with non-POD group, POD was more likely to occur in patients who were female, older than 65 years, and education level less than 9 years, had history of alcohol abuse and smoking, had history of hypertension, diabetes and 
stroke, ASA physical status III - IV, supra-tentorial lesions, intraoperative use of steroid, intraoperative transfusion, and anesthesia duration $>6$ hours, as well as had GCS $<9$, metabolic acidosis, use of PCIA, and positioning of neurosurgical drainage tube at ICU admission (Table 1). 
Table 1

Univariate analyses for demographics, pre-operative data, anaesthesia-related and intra-operative data (categorical variables).

\begin{tabular}{|c|c|c|c|c|}
\hline Characteristic & $\begin{array}{l}\text { Delirium } \\
(n=157)\end{array}$ & $\begin{array}{l}\text { No Delirium } \\
(n=643)\end{array}$ & $\chi^{2}$ & $P$ value \\
\hline Gender of Female & $80(51.0 \%)$ & $392(61.0 \%)$ & 6.086 & 0.022 \\
\hline Age $>65$ years & $40(25.5 \%)$ & $49(7.6 \%)$ & 40.697 & $<0.001$ \\
\hline Education level $<9$ years & $51(32.5 \%)$ & $89(13.8 \%)$ & 30.377 & $<0.001$ \\
\hline History of alcohol abuse & $46(29.3 \%)$ & $121(18.8 \%)$ & 8.393 & 0.004 \\
\hline History of smoking & $64(40.8 \%)$ & $122(19.0 \%)$ & 33.579 & $<0.001$ \\
\hline History of hypertension & $51(32.5 \%)$ & $140(21.8 \%)$ & 7.966 & 0.005 \\
\hline History of coronary artery disease & $9(5.7 \%)$ & $24(3.7 \%)$ & 1.276 & 0.259 \\
\hline History of diabetes & $18(11.5 \%)$ & $32(5.0 \%)$ & 9.066 & 0.003 \\
\hline History of stroke & $13(8.3 \%)$ & $27(4.2 \%)$ & 4.425 & 0.035 \\
\hline Preoperative of hydrocephalus & $8(5.1 \%)$ & $34(5.3 \%)$ & 0.009 & 0.923 \\
\hline History of epilepsy & $17(10.8 \%)$ & $42(6.5 \%)$ & 3.409 & 0.065 \\
\hline Supra-tentorial lesions & $93(59.2 \%)$ & $217(33.7 \%)$ & 34.538 & $<0.001$ \\
\hline ASA physical status (III - IV) & $16(10.2 \%)$ & $14(2.2 \%)$ & 22.452 & $<0.001$ \\
\hline TIVA & $33(21.0 \%)$ & $153(23.8 \%)$ & 0.278 & 0.462 \\
\hline Intraoperative use of dexmedetomidine & $19(12.1 \%)$ & $91(14.2 \%)$ & 0.447 & 0.504 \\
\hline Intraoperative use of steroid & $41(26.1 \%)$ & $108(16.8 \%)$ & 7.230 & 0.007 \\
\hline Intraoperative transfusion & $61(38.9 \%)$ & $134(20.8 \%)$ & 22.213 & $<0.001$ \\
\hline Anaesthesia duration > $360 \mathrm{~min}$ & $101(64.3 \%)$ & $294(45.7 \%)$ & 17.480 & $<0.001$ \\
\hline GCS $<9$ at ICU admission & $93(59.2 \%)$ & $136(21.2)$ & 89.584 & $<0.001$ \\
\hline Anemia & $11(7.0 \%)$ & $42(6.5 \%)$ & 0.046 & 0.830 \\
\hline Hypoalbuminemia & $62(39.5 \%)$ & $159(24.7 \%)$ & 13.755 & $<0.001$ \\
\hline Metabolic acidosis & $32(20.4 \%)$ & $15(2.3 \%)$ & 74.341 & $<0.001$ \\
\hline Hyponatremia & $34(21.7 \%)$ & $161(25.0 \%)$ & 0.783 & 0.376 \\
\hline
\end{tabular}

Data are shown as $\mathrm{N}(\%)$. ASA, American Society of anesthesiologists; TIVA, total intravenous anaesthesia; GCS, Glasgow coma score; ICU, intensive care unit; PCIA, patient-controlled intravenous analgesia. 


\begin{tabular}{|lllll|}
\hline Characteristic & $\begin{array}{l}\text { Delirium } \\
(\mathbf{n = 1 5 7 )}\end{array}$ & $\begin{array}{l}\text { No Delirium } \\
(\mathbf{n = 6 4 3 )}\end{array}$ & $\chi^{2}$ & Pvalue \\
\hline PCIA & $43(27.4 \%)$ & $286(44.5 \%)$ & 15.223 & $<0.001$ \\
\hline positioning of neurosurgical drainage tube & $70(44.6 \%)$ & $125(19.4 \%)$ & 43.286 & $<0.001$ \\
\hline $\begin{array}{l}\text { Data are shown as N (\%). ASA, American Society of anesthesiologists; TIVA, total intravenous } \\
\text { anaesthesia; GCS, Glasgow coma score; ICU, intensive care unit; PCIA, patient-controlled intravenous } \\
\text { analgesia. }\end{array}$ & & & \\
\hline
\end{tabular}

By univariate analysis, we selected 17 candidate predictors that met both the clinical and statistical criteria of $p<0.10$ into a multivariable logistic regression analysis. Those candidate predictors are gender (female), age $>65$ years, education level $<9$ years, history of alcohol abuse, history of smoking, history of hypertension, history of diabetes, history of stroke, ASA physical status III - IV, supra-tentorial lesions, intraoperative use of steroid, intraoperative transfusion, anesthesia duration $>6$ hours, GCS $<9$ at ICU admission, metabolic acidosis, use of PCIA, and positioning of neurosurgical drainage tube.

In the stepwise backward logistic regression analysis for the final model, the 9 dichotomized variables that best predicted outcome were: age $>65$ years $(\mathrm{OR}=3.336 ; 95 \% \mathrm{Cl}, 1.765-6.305)$, education level $<9$ years $(\mathrm{OR}=2.528 ; 95 \% \mathrm{Cl}, 1.446-4.419)$, history of smoking $(\mathrm{OR}=2.582 ; 95 \% \mathrm{Cl}, 1.611-4.140)$, history of diabetes $(\mathrm{OR}=2.541,95 \% \mathrm{Cl}, 1.201-5.377)$, supra-tentorial lesions $(\mathrm{OR}=3.424 ; 95 \% \mathrm{Cl}, 2.021-5.802)$, anesthesia duration $>360 \mathrm{~min}(\mathrm{OR}=1.686,95 \% \mathrm{Cl}, 1.062-2.674), \mathrm{GCS}<9$ at ICU admission $(\mathrm{OR}=6.059$, $95 \% \mathrm{Cl}, 3.789-9.690)$, metabolic acidosis ( $\mathrm{OR}=13.903,95 \% \mathrm{Cl}, 6.248-30.938)$, and positioning of neurosurgical drainage tube $(\mathrm{OR}=1.924 ; 95 \% \mathrm{Cl}, 1.132-3.269)$ (Table 2$)$. 
Table 2

Multivariate logistic regression model with potential predictors for according to bivariate analysis

\begin{tabular}{|c|c|c|c|c|}
\hline Variables & $\begin{array}{l}\text { Regression } \\
\text { Coefficient }\end{array}$ & $\begin{array}{l}\text { Adjusted odds } \\
\text { ratio }\end{array}$ & $(95 \% \mathrm{Cl})$ & $\begin{array}{l}P \\
\text { value }\end{array}$ \\
\hline Gender of Female & -0.043 & 0.958 & $\begin{array}{l}0.541- \\
1.696\end{array}$ & 0.883 \\
\hline Age $>65$ years & 1.205 & 3.336 & $\begin{array}{l}1.765- \\
6.305\end{array}$ & $<$. \\
\hline Education level $<9$ years & 0.927 & 2.528 & $\begin{array}{l}1.446- \\
4.419\end{array}$ & 0.001 \\
\hline History of alcohol abuse & -0.417 & 0.659 & $\begin{array}{l}0.335- \\
1.297\end{array}$ & 0.227 \\
\hline History of smoking & 0.949 & 2.582 & $\begin{array}{l}1.611- \\
4.140\end{array}$ & $\hat{0}_{0.001}$ \\
\hline History of hypertension & 0.255 & 1.290 & $\begin{array}{l}0.764- \\
2.179\end{array}$ & 0.341 \\
\hline History of diabetes & 0.933 & 2.541 & $\begin{array}{l}1.201- \\
5.377\end{array}$ & 0.015 \\
\hline History of stroke & 0.378 & 1.430 & $\begin{array}{l}0.557- \\
3.671\end{array}$ & 0.457 \\
\hline History of epilepsy & 0.238 & 1.269 & $\begin{array}{l}0.574- \\
2.802\end{array}$ & 0.556 \\
\hline Supra-tentorial lesions & 1.249 & 3.488 & $\begin{array}{l}2.033- \\
5.981\end{array}$ & $\hat{0}_{0.001}$ \\
\hline ASA physical status (III - IV) & 0.769 & 2.158 & $\begin{array}{l}0.814- \\
5.721\end{array}$ & 0.122 \\
\hline Intraoperative use of steroid & -0.095 & 0.909 & $\begin{array}{l}0.524- \\
1.579\end{array}$ & 0.736 \\
\hline Intraoperative transfusion & 0.211 & 1.235 & $\begin{array}{l}0.729- \\
2.091\end{array}$ & 0.433 \\
\hline Anaesthesia duration $>360 \mathrm{~min}$ & 0.522 & 1.686 & $\begin{array}{l}1.062- \\
2.674\end{array}$ & 0.027 \\
\hline GCS $<9$ at ICU admission & 1.802 & 6.059 & $\begin{array}{l}3.789- \\
9.690\end{array}$ & $\begin{array}{l}< \\
0.001\end{array}$ \\
\hline Hypoalbuminemia & 0.239 & 1.270 & $\begin{array}{l}0.784- \\
2.058\end{array}$ & 0.331 \\
\hline
\end{tabular}

Nagelkerke $\mathrm{R}^{2}=0.436$, Hosmer-Lemeshow goodness-of-fit test $(\chi 2=6.910$, degrees of freedom $=8, P$ $=0.546) ; A S A$, American Society of anesthesiologists; GCS, Glasgow coma score; ICU, intensive care unit; PCIA, patient-controlled intravenous analgesia. 


\begin{tabular}{|lllll|}
\hline Variables & $\begin{array}{l}\text { Regression } \\
\text { Coefficient }\end{array}$ & $\begin{array}{l}\text { Adjusted odds } \\
\text { ratio }\end{array}$ & $(95 \%$ Cl) & $\begin{array}{l}P \\
\text { value }\end{array}$ \\
\hline Metabolic acidosis & 2.632 & 13.903 & $\begin{array}{l}6.248- \\
30.938\end{array}$ & 0.001 \\
\hline PCIA & -0.286 & 0.751 & $\begin{array}{l}0.461- \\
1.223\end{array}$ & 0.250 \\
\hline $\begin{array}{l}\text { positioning of neurosurgical } \\
\text { drainage tube }\end{array}$ & 0.654 & 1.924 & $\begin{array}{l}1.132- \\
3.269\end{array}$ & 0.016 \\
\hline Constants & -4.148 & 0.016 & - & 0.001 \\
\hline $\begin{array}{l}\text { Nagelkerke } \mathrm{R}^{2}=0.436, \text { Hosmer-Lemeshow goodness-of-fit test }(\chi 2=6.910, \text { degrees of freedom }=8, P \\
=0.546) ; A S A, \text { American Society of anesthesiologists; GCS, Glasgow coma score; ICU, intensive care } \\
\text { unit; PCIA, patient-controlled intravenous analgesia. }\end{array}$ & \\
\hline
\end{tabular}

The formula for our prediction model are as followed: Risk of POD $=1 /[1+\exp -(-4.148+1.206 \times$ age $>65$ years $+0.927 \times$ education level $<9$ years $+0.949 \times$ history of smoking $+0.933 \times$ history of diabetes + $1.231 \times$ supra-tentorial lesions $+0.522 \times$ anesthesia duration $>360 \mathrm{~min}+1.802 \times \mathrm{GCS}<9$ at ICU admission $+2.632 \times$ metabolic acidosis $+0.654 \times$ positioning of neurosurgical drainage tube)]. According to the $\beta$ coefficients for each variable, values were assigned as follows: 1 for age $>65$ years, 1 for education level < 9 years, 1 for history of smoking, 1 for history of diabetes, 1 for supra-tentorial lesions, 0.5 for anesthesia duration $>360 \mathrm{~min}, 1.5$ for GCS $<9$ at ICU admission, 2.5 for metabolic acidosis, and 0.5 for positioning of neurosurgical drainage tube (Fig. 1 \& Additional file: Table S1). The comparison table of total score and probability of POD is showed in Additional file: Table S2.

\section{Score Performance And Internal Validation}

The final model showed good calibration (Fig. 2C) using the Hosmer-Lemeshow goodness-of-fit test $(\chi 2=$ 6.910, degrees of freedom $=7, p=0.546$ ) and good discrimination (AUROC $=0.865,95 \% \mathrm{Cl} 0.835-0.895$; Fig. $2 \mathrm{~A}$ ) without collinearity (variance inflation factor $=1.362$ ). The sensitivity of the model was 0.78 , and the specificity was 0.82 . Combined with its sensitivity and specificity, 0.59 was defined as the cut-off point of the model. On performing bootstrapping with 200 repetitions for internal validation, no significant differences were found regarding the model, the variables, or their regression coefficients. The performance of the prediction model was further confirmed by bootstrap internal validation, in which the AUROC was 0.851 (95\% Cl 0.791-0.912) (Fig. 2B), and calibration plot showed pretty fitting effect (Fig. 2D).

A cut-off value of 2 was identified to distinguish the high or low risk of POD. In patients with E-PREPODNS score $\leq 2$, the POD rate was $6 \%$. However, in those with E-PREPOD-NS score $>2$, the POD rate was $46.1 \%$ (Additional file: Table S3). In addition, to evaluate the ability of the model to predict increasing 
rates of POD, categorisation using cutoffs of 3 and 6 produced three groups with clearly different incidences of POD in neurosurgical patients (Fig. 3).

\section{Discussion}

In the present study, we developed and internally validated a practical prediction model for POD in neurosurgical patients admitted to the ICU after elective craniotomy, consisting of 9 clinical factors: age, education level, history of smoking, history of diabetes, supra-tentorial lesions, anaesthesia duration, GCS at ICU admission, metabolic acidosis, and positioning of neurosurgical drainage tubes. For ease of application, we turned the risk prediction model into a risk prediction score with a total score of 10 points. E-PREPOD-NS score is the first simplified clinical score for early predicting POD in neurosurgical patients in ICU that can be used at the time of ICU admission and with good discriminative power for identifying patients at risk of POD.

Clinical studies have shown that POD is associated with high morbidity and mortality (Franco et al. 2001; Abelha et al. 2013; Gleason et al. 2015; Evered et al. 2018). 30-40\% of delirium can be preventable (Siddiqi et al. 2006). When preventive measures are restricted to high-risk patients, the number of patients who will be unnecessarily exposed to potential harmful side effects are likely less. Therefore, developing and routine use of the risk prediction model for POD may facilitate early recognition and identification of those patients at high risk of POD who may benefit the most from POD preventive measures (Siddiqi 2016). More importantly, the use of delirium risk stratification to target high-risk patients makes wider implementation of multicomponent interventions aimed at preventing delirium more feasible when resources are limited (Siddiqi 2016). At present, two delirium model have been developed and validated in the multinational cohorts for use in adults patients admitted to the mixed ICU (van den Boogaard et al. 2012; van den Boogaard et al. 2014; Wassenaar et al. 2015). One prediction model for delirium in ICU patients (PRE-DELIRIC model) can reliably predict ICU patients' risk of delirium using ten predictors obtained within the first $24 \mathrm{~h}$ of ICU admission (van den Boogaard et al. 2014). Given that up to $25 \%$ of delirium might occur within the first $24 \mathrm{~h}$ of ICU admission (Ely et al. 2004; Serafim et al. 2012), and delirium prevention measures should be deployed as early as possible. Therefore, an early prediction model (E-PRE-DELIRIC model) was developed to predict the risk of delirium at the moment that a patient is admitted to the ICU (Wassenaar et al. 2015). However, neurosurgical patients are the one of most fragile patients who require maximum preventive efforts (van den Boogaard et al. 2012), and a prediction model for POD specific to this vulnerable population may provide unique insights. The current prediction models of neurosurgical patients are aimed at Parkinson's patients after deep brain stimulation surgery (Zhan et al. 2020), patients undergoing cerebrovascular surgery (Harasawa, Mizuno 2014) and glioblastoma patients (Flanigan et al. 2018). Only one model for mixed neurosurgical patients in ICU have developed by Wang (Wang et al. 2020b), but it did not include neurosurgery-specific factors and cannot early predict POD at the time of ICU admission. In the present study, we developed a prediction model for POD in neurosurgical patients admitted to ICU involving 7 clinical and laboratory variables which were already reported in the previous literatures (Aldecoa et al. 2017) and 2 neurosurgery-specific factors. In addition, in order to adopt preventive measures as early as possible, we develop and validate our model 
based on data available at ICU admission. More importantly, the score is easy to calculate and can be applied to almost all ICUs, even in resource-limited settings.

According to previous reviews of POD prediction (Aldecoa et al. 2017), our study identified advanced age, education level, history of smoking, history of diabetes, anesthesia duration, GCS $<9$ at ICU admission and metabolic acidosis as leading risk factors. In our analysis, some variables were previously reported and had high significance in univariate analysis (history of alcohol abuse, history of hypertension, history of stroke, ASA physical status and intraoperative transfusion), but were not selected into the final model. This means there may be some indirect associations between these variables and independent predictors. We still need more researches about these variables.

Two neurosurgical-related risk factors were identified in our prediction model: supra-tentorial lesions and positioning of neurosurgical drainage tubes. Investigations observed that the attentional networks model (including alerting, orienting and executive control) (Petersen et al. 2012) involved an extensive supratentorial anatomical distribution such as frontal and parietal regions with interconnecting pathways, dorsolateral prefrontal cortex, anterior cingulate cortex, and the thalamus with connections to the frontal lobe, the superior parietal lobe and the superior temporal lobe (Corbetta et al. 2002; Posner et al. 2006; Petersen et al. 2012). Therefore, cognitive function may be affected by supra-tentorial lesions which may explain the increased risk of POD in the current study. In addition, we found that positioning of neurosurgical drainage tubes was an independent risk factor for POD. We considered that the presence of intracranial drainage, the use of sedative drugs, and the necessity of physical restraint are interlocking. Several studies have demonstrated physical (Kubota et al. 2018) and chemical restraint (Kok et al. 2018; Cozowicz et al. 2020) predisposed patients to a greater risk of POD. Therefore, for postoperative neurosurgical patients with intracranial drainage, although early activities are restricted, the use of an analgesic-based sedation protocol or the choice of a more suitable sedative drug for sedation may help to prevent POD.

Based on the accuracy of ROC risk stratification, patients with total scores less than or equal to 2 was associated with low risk of POD, otherwise with high risk. ROC analysis further revealed total scores greater than 5.5 yielded a hundred percent specificity, which indicated patients with total scores greater than or equal to 6 were major concerns in the high risk group. It was noteworthy that there was have around $50 \%$ probability of POD occurred in patients with ranging 2.5 to 5.5 according to the corresponding probabilities calculation of the model. Therefore, the use of the E-PREPOD-NS model to identify and subsequently preventively treat moderate and high risk patients could offer a great contribution to perioperative intensive care practice and ensure efficient use of medical resources to provide to the only patients at risk.

There are several strengths of our study. Firstly, we included very comprehensive candidate risk factors in our study, including those risk factors reported in previous studies and those unreported neurosurgeryspecific factors. All of them are easily to obtain from medical history and routine clinical examinations, and only 9 variables in the final model. Therefore, our risk scores might be convenient for clinicians to 
apply and simple to calculate. Secondly, the patients who were enrolled in our cohort had been admitted after elective craniotomy with various types of disease that is similar to real-life situation. Delirium occurs in patients with traumatic brain injury (TBI) and stroke might be more closely to critical illness rather than surgical stress. Therefore, TBI and stroke patients were excluded in our cohort. Thirdly, multicollinearity was not found among the 9 predictors which were tested by variance inflation factors.

However, our study also has several limitations. The primary limitation is that this is a single-center study. We only conducted internally verification and did not perform externally verification. Therefore, our model might be limited for widely generalizing in other races and regions. Secondly, recent studies emphasized the importance of biomarkers (Khan et al. 2020) and intra-operative electroencephalogram signatures (Fritz et al. 2020) to predict POD. However, for ease of application, we did not include biomarkers and intra-operative electroencephalogram signatures because they are not yet widely used clinically. Thirdly, the latest definition of POD is that delirium occurs in hospital up to 1 week post-operation or until discharge (whichever occurs first) (Evered et al. 2018). Nevertheless, only POD on postoperative 1 to 3 were available in our previous dataset. Finally, in order to facilitate statistics, we represent our variables as binary variables, and the severity of each variable is not taken into account.

\section{Conclusions}

In conclusion, we built a practical model with 9 clinical factors to predict POD for neurosurgical patients admitted to the ICU after elective craniotomy. It will be necessary to externally validate our results in future studies to investigate how this score model affects the prevention of POD in clinical practice.

\section{Abbreviations}

E-PREPOD-NS: early prediction model for postoperative delirium in neurosurgical patients admitted to the ICU after elective craniotomy; POD: postoperative delirium; ICU: intensive care unit; pNCD: postoperative neurocognitive dysfunctions; CAM-ICU: Confusion Assessment Method for the ICU; RASS: Richmond Agitation-Sedation Scale; GCS: Glasgow Coma Scale; ASA: American Society of Anesthesiologists; TIVA: total intravenous anaesthesia; PCIA: patient-controlled intravenous analgesia; EVD: external ventricular drainage; IQR: interquartile range; ORs: odds ratios; Cls: confidence intervals; AUROC: area under the receiver operator curve; SE: sensitivity; SP: specificity; PPV: positive predictive value; NPV: negative predictive value; LR: positive likelihood ratio; LR: negative likelihood ratio; TBI: traumatic brain injury

\section{Declarations}

Acknowledgements

We thank all the staffs in ICUs and department of neurosurgery to participate in this study.

Authors' contributions 
$\mathrm{JZ}$ and $\mathrm{HH}$ conceived and managed the study. $\mathrm{HH}, \mathrm{GZ}$ and $\mathrm{HL}$ joined in study design, data collection, data analysis, manuscript preparation and revision. CW, YW, XS, JC, GC and MX participated in data collection. All authors read and approved the final manuscript.

\section{Funding}

This work was supported by the National Natural Science Foundation of China (81801042), Beijing Hospital Authority Youth Programme (20190504) and Beijing Outstanding Talent Training Foundation, Youth Backbone Individual Project (2018000021469G230). The funds had no role in the study design, data collection and analysis, decision to publish, or manuscript preparation.

Availability of data and materials

The datasets analyzed during the current study available from the corresponding author on reasonable request.

Ethics approval and consent to participate

The Institutional Review Board of the Beijing Tiantan hospital approved the study. Informed consent was obtained from patients or their family members.

\section{Consent for publication}

All authors have reviewed and approved the manuscript for publication

\section{Conflicts of interest}

The authors declare that they have no competing interests.

\section{References}

1. Abelha FJ, Luis C, Veiga D, Parente D, Fernandes V, Santos $P$, et al. Outcome and quality of life in patients with postoperative delirium during an ICU stay following major surgery. Crit Care. 2013;17:R257.

2. Aldecoa C, Bettelli G, Bilotta F, Sanders RD, Audisio R, Borozdina A, et al. European Society of Anaesthesiology evidence-based and consensus-based guideline on postoperative delirium. Eur $\mathrm{J}$ Anaesthesiol. 2017;34:192-214.

3. Berian JR, Zhou L, Russell MM, Hornor MA, Cohen ME, Finlayson E, et al. Postoperative Delirium as a Target for Surgical Quality Improvement. Ann Surg. 2018;268:93-9.

4. Bohner $\mathrm{H}$, Hummel TC, Habel U, Miller $\mathrm{C}$, Reinbott $\mathrm{S}$, Yang Q, et al. Predicting delirium after vascular surgery: a model based on pre- and intraoperative data. Ann Surg. 2003;238:149-56.

5. Corbetta M, Shulman GL. Control of goal-directed and stimulus-driven attention in the brain. Nat Rev Neurosci. 2002;3:201-15. 
6. Cozowicz C, Memtsoudis SG, Poeran J. Risk factors for postoperative delirium in patients undergoing lower extremity joint arthroplasty: a retrospective population-based cohort study. Reg Anesth Pain Med. 2020;46:94-5.

7. Ely EW, Shintani A, Truman B, Speroff T, Gordon SM, Harrell FE Jr, et al. Delirium as a predictor of mortality in mechanically ventilated patients in the intensive care unit. JAMA. 2004;291:1753-62.

8. Evered L, Silbert B, Knopman DS, Scott DA, DeKosky ST, Rasmussen LS, et al. Recommendations for the nomenclature of cognitive change associated with anaesthesia and surgery-2018. Br J Anaesth. 2018;121:1005-12.

9. Flanigan PM, Jahangiri A, Weinstein D, Dayani F, Chandra A, Kanungo I, et al. Postoperative Delirium in Glioblastoma Patients: Risk Factors and Prognostic Implications. Neurosurgery. 2018;83:1161-72.

10. Franco K, Litaker D, Locala J, Bronson D. The cost of delirium in the surgical patient. Psychosomatics. 2001;42:68-73.

11. Fritz BA, King CR, Ben Abdallah A, Lin N, Mickle AM, Budelier TP, et al. Preoperative Cognitive Abnormality, Intraoperative Electroencephalogram Suppression, and Postoperative Delirium: A Mediation Analysis. Anesthesiology. 2020;132:1458-68.

12. Gleason LJ, Schmitt EM, Kosar CM, Tabloski P, Saczynski JS, Robinson T, et al. Effect of Delirium and Other Major Complications on Outcomes After Elective Surgery in Older Adults. JAMA Surg. 2015;150:1134-40.

13. Harasawa N, Mizuno T. A novel scale predicting postoperative delirium (POD) in patients undergoing cerebrovascular surgery. Arch Gerontol Geriatr. 2014;59:264-71.

14. Inouye SK, Westendorp RG, Saczynski JS. Delirium in elderly people. Lancet. 2014;383:911-22.

15. Kadane JB, Lazar NA. Methods and Criteria for Model Selection. J Am Stat Assoc. 2004;99:279-90.

16. Khan BA, Perkins AJ, Prasad NK, Shekhar A, Campbell NL, Gao S, et al. Biomarkers of Delirium Duration and Delirium Severity in the ICU. Crit Care Med. 2020;48:353-61.

17. Kim EM, Li G, Kim M. Development of a Risk Score to Predict Postoperative Delirium in Patients With Hip Fracture. Anesth Analg. 2020;130:79-86.

18. Kim MY, Park UJ, Kim HT, Cho WH. DELirium Prediction Based on Hospital Information (Delphi) in General Surgery Patients. Medicine. 2016;95:e3072.

19. Kok L, Slooter AJ, Hillegers MH, van Dijk D, Veldhuijzen DS. Benzodiazepine Use and Neuropsychiatric Outcomes in the ICU: A Systematic Review. Crit Care Med. 2018;46:1673-80.

20. Kubota K, Suzuki A, Ohde S, Yamada U, Hosaka T, Okuno F, et al. Age is the Most Significantly Associated Risk Factor With the Development of Delirium in Patients Hospitalized for More Than Five Days in Surgical Wards: Retrospective Cohort Study. Ann Surg. 2018;267:874-7.

21. Omar RZ. Clinical Prediction Models: A Practical Approach to Development, Validation and Updating by STEYERBERG. E W Biometrics. 2010;66:661-2.

22. Petersen SE, Posner MI. The attention system of the human brain: 20 years after. Annu Rev Neurosci. 2012;35:73-89. 
23. Posner MI, Sheese BE, Odludas Y, Tang Y. Analyzing and shaping human attentional networks. Neural Netw. 2006;19:1422-9.

24. Serafim RB, Dutra MF, Saddy F, Tura B, de Castro JE, Villarinho LC, et al. Delirium in postoperative nonventilated intensive care patients: risk factors and outcomes. Ann Intensive Care. 2012;2:51.

25. Sessler CN, Gosnell MS, Grap MJ, Brophy GM, O'Neal PV, Keane KA, et al. The Richmond AgitationSedation Scale: validity and reliability in adult intensive care unit patients. Am J Respir Crit Care Med. 2002;166:1338-44.

26. Siddiqi N. Predicting delirium: time to use delirium risk scores in routine practice? Age Ageing. 2016;45:9-10.

27. Siddiqi N, House AO, Holmes JD. Occurrence and outcome of delirium in medical in-patients: a systematic literature review. Age Ageing. 2006;35:350-64.

28. Steyerberg EW, Vickers AJ, Cook NR, Gerds T, Gonen M, Obuchowski N, et al. Assessing the performance of prediction models: a framework for traditional and novel measures. Epidemiology. 2010;21:128-38.

29. van den Boogaard M, Pickkers P, Slooter AJ, Kuiper MA, Spronk PE, van der Voort PH, et al. Development and validation of PRE-DELIRIC (PREdiction of DELIRium in ICu patients) delirium prediction model for intensive care patients: observational multicentre study. BMJ. 2012;344:e420.

30. van den Boogaard M, Schoonhoven L, Maseda E, Plowright C, Jones C, Luetz A, et al. Recalibration of the delirium prediction model for ICU patients (PRE-DELIRIC): a multinational observational study. Intensive Care Med. 2014;40:361-9.

31. Wang C, Wu Y, Yue P, Ely EW, Huang J, Yang X, et al. Delirium assessment using Confusion Assessment Method for the Intensive Care Unit in Chinese critically ill patients. J Crit Care. 2013;28:223-9.

32. Wang CM, Huang HW, Wang YM, He X, Sun XM, Zhou YM, et al. Incidence and risk factors of postoperative delirium in patients admitted to the ICU after elective intracranial surgery: $A$ prospective cohort study. Eur J Anaesthesiol. 2020a;37:14-24.

33. Wang J, Ji Y, Wang N, Chen W, Bao Y, Qin Q, et al. Establishment and validation of a delirium prediction model for neurosurgery patients in intensive care. Int J Nurs Pract. 2020b;26:e12818.

34. Wassenaar A, van den Boogaard M, van Achterberg T, Slooter AJ, Kuiper MA, Hoogendoorn ME, et al. Multinational development and validation of an early prediction model for delirium in ICU patients. Intensive Care Med. 2015;41:1048-56.

35. Zhan L, Wang XQ, Zhang LX. Nomogram Model for Predicting Risk of Postoperative Delirium After Deep Brain Stimulation Surgery in Patients Older Than 50 Years with Parkinson Disease. World Neurosurg. 2020;139:e127-35.

\section{Figures}




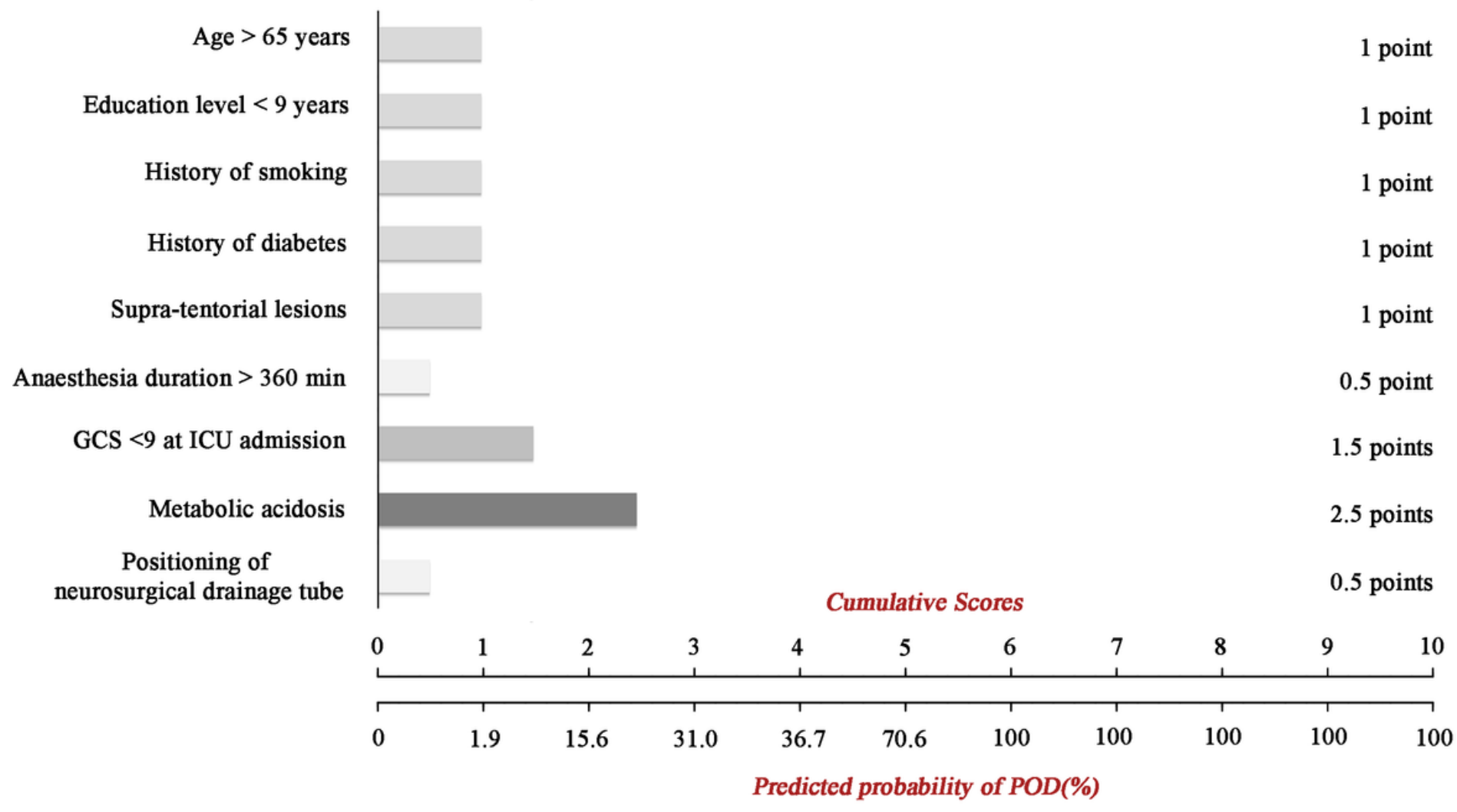

\section{Figure 1}

Predictive score model for POD 
A

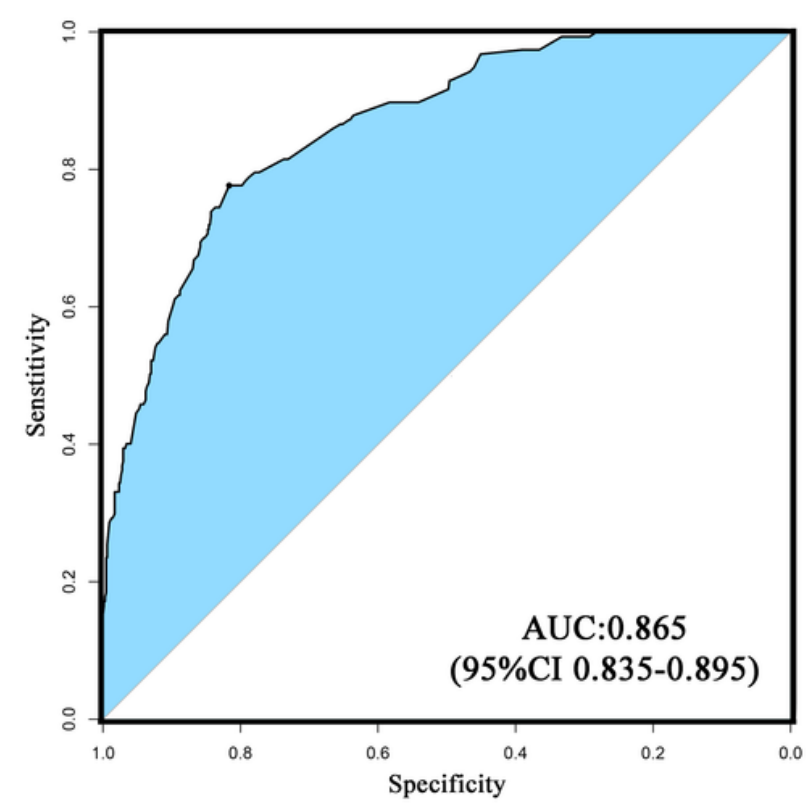

C

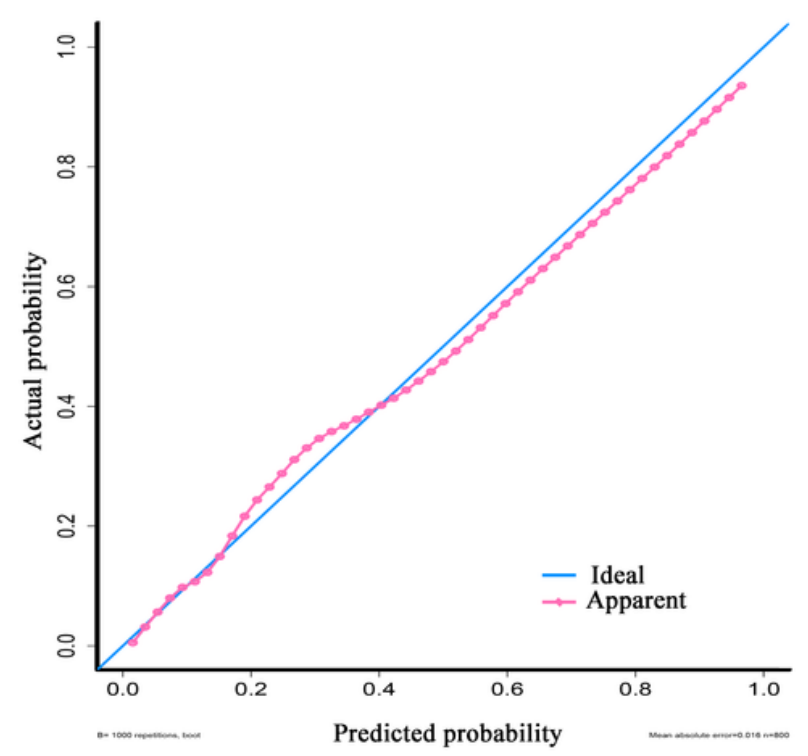

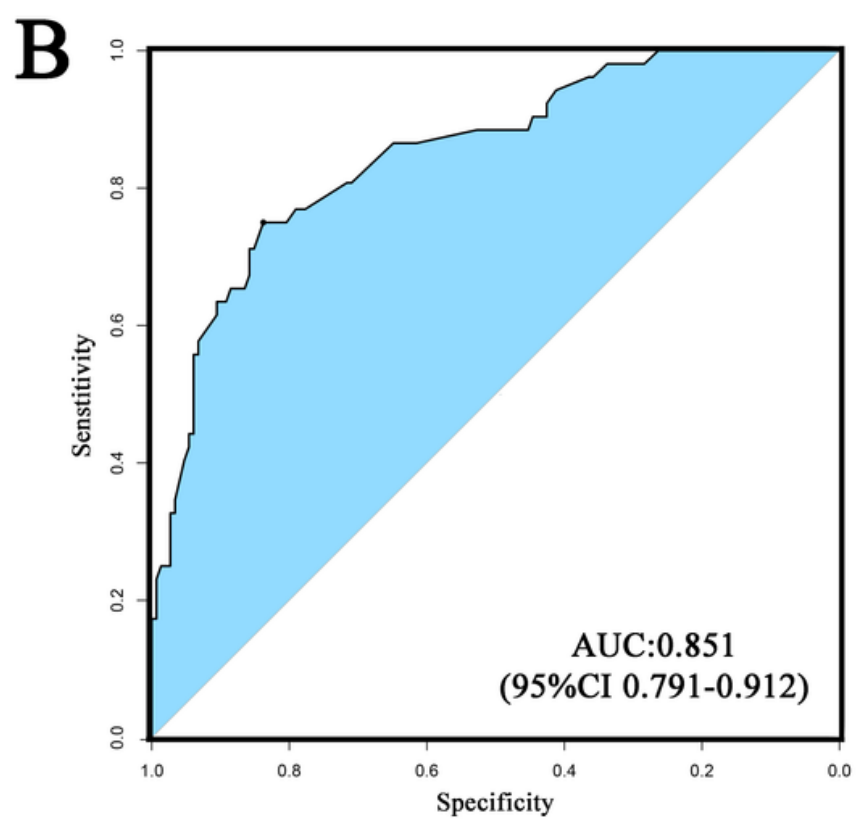

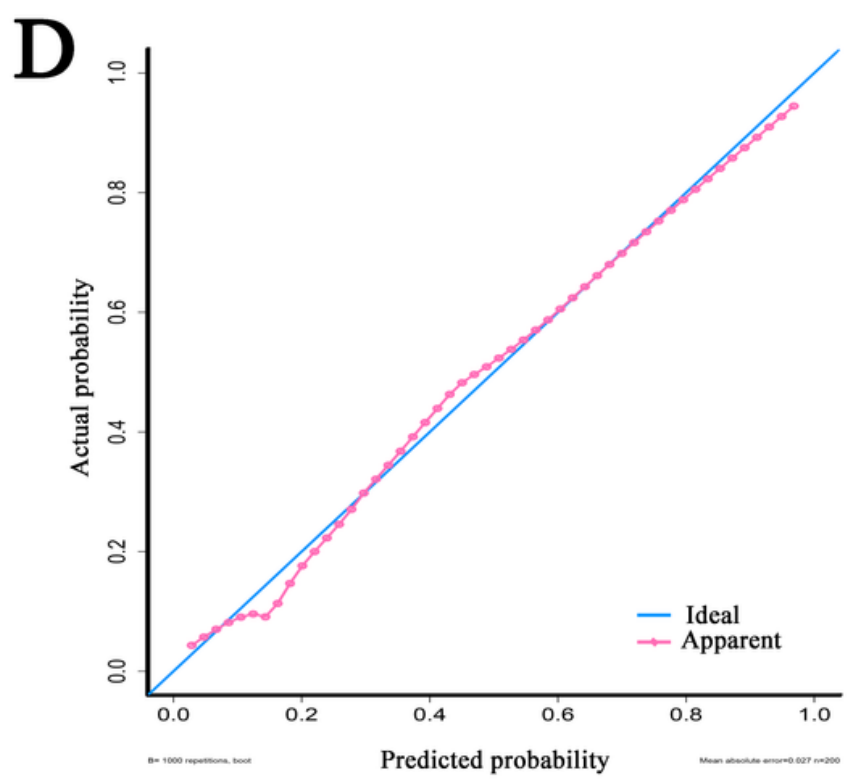

\section{Figure 2}

Receiver-operating characteristic $(\mathrm{ROC})$ curve and calibration curve of the predictive model. (A-B) ROC curves of the model in the training cohort and validation cohort. (C-D) Calibration curves of the model in the training cohort and validation cohort. 


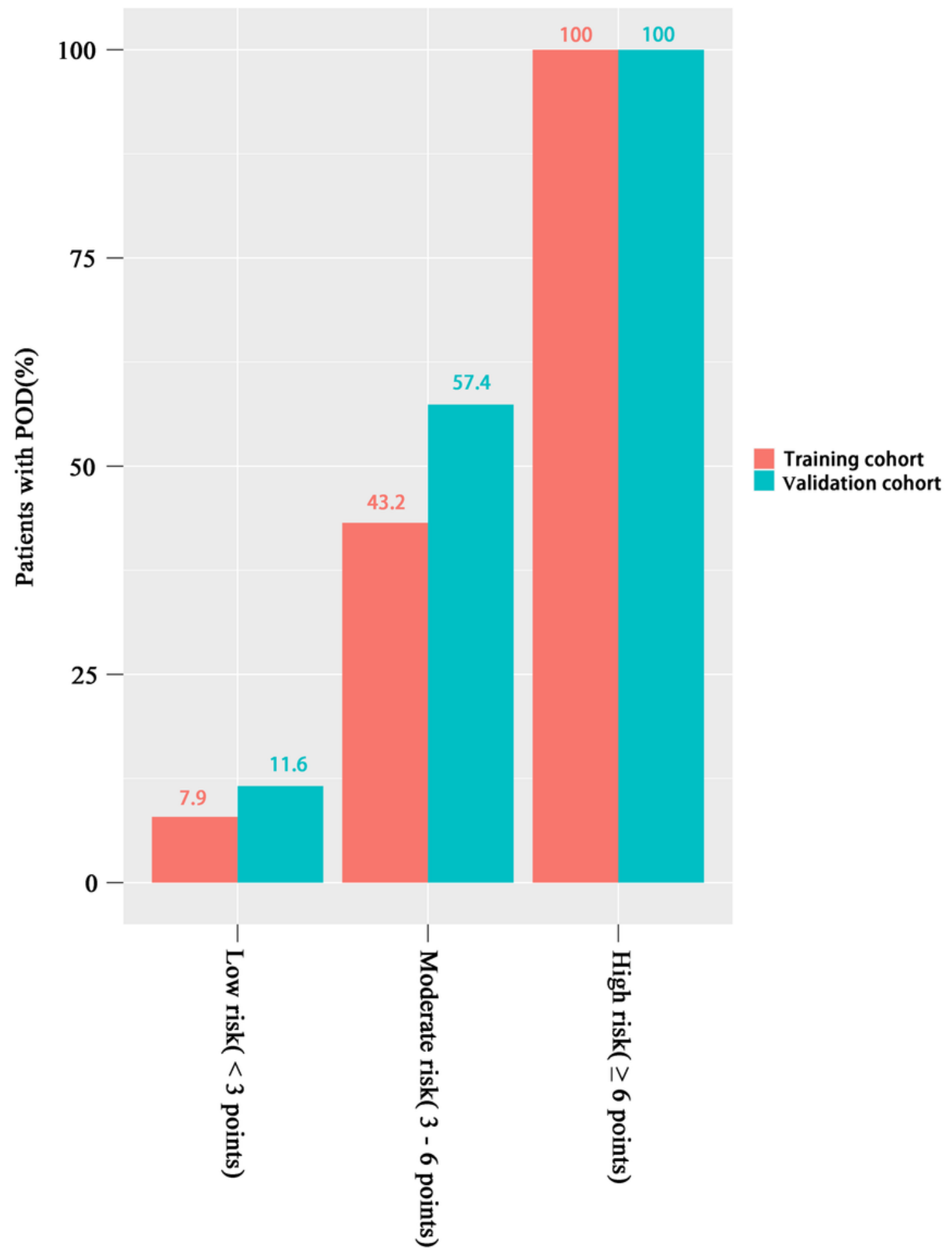

Figure 3

Column plot demonstrating probabilities of delirium after neurosurgical interventions according to the predictive scores of the POD over the tertiles.

\section{Supplementary Files}


This is a list of supplementary files associated with this preprint. Click to download.

- Additionalflie.docx 